\title{
A Novel Technique for Umbilical Reconstruction Using Four Transposition Flaps
}

\author{
Yoon Soo Kim, Eon Ju Park, \\ Hyung Suk Yi, Jin Hyung Park \\ Department of Plastic and Reconstructive \\ Surgery, Kosin University College of \\ Medicine, Busan, Korea
}

No potential conflict of interest relevant to this article was reported.
The umbilicus is of paramount aesthetic importance for the abdomen, and its absence can be psychologically distressing to patients for cosmetic reasons. An aestheticallypleasing umbilicus tends to be small and vertically oriented in nature, with superior hooding and shadow, inferior retraction and slope, and a position at the topmost level of the iliac crest. A 42-year-old woman had undergone delayed breast reconstruction using a transverse rectus abdominis myocutaneous (TRAM) flap. Unfortunately, the patient developed umbilical necrosis following surgery. She underwent complete surgical debridement, which resulted in a large vertical scar. Delayed umbilical reconstruction was performed with four transposition flaps. The patient underwent follow-up at 11 months postoperatively, and the umbilicus had a satisfactory appearance. This case shows that using four transposition flaps can yield sufficient depth and an aesthetically pleasing shape for the umbilicus.

Keywords Abdominoplasty, Surgical flaps, Umbilicus

\section{INTRODUCTION}

The umbilicus is at the center of the abdomen and has important aesthetic value. A natural and aesthetic umbilicus is small in size and has an inverted $\mathrm{T}$ or vertical shape with superior hooding. An absence of the umbilicus or a change in its shape can be psychologically distressing for the patient because of its cosmetic implications. To create a natural and aesthetically pleasing umbilicus, various methods, such as the use of local flaps, the purse-string suture, and cartilage grafts have been introduced [1-3], but no optimal method of umbilical reconstruction has yet been developed.

A natural abdominal wall periumbilical concavity is necessary for a smooth transition between the abdominal wall and the umbilicus with an inconspicuous scar [4]. In this study, we used the local flap method of umbilical reconstruction to design four transpo-

Received: Feb 24, 2016 Revised: May 2, 2016 Accepted: May 10, 2016 Correspondence: Jin Hyung Park Department of Plastic and Reconstructive Surgery, Kosin University College of Medicine, 262 Gamcheon-ro, Seo-gu, Busan 49267, Korea. E-mail: atreyue@naver.com

Copyright () 2016 The Korean Society for Aesthetic Plastic Surgery.

This is an Open Access article distributed under the terms of the Creative Commons Attribution Non-Commercial License (http://creativecommons.org/licenses/by-nc/4.0/) which permits unrestricted non-commercial use, distribution, and reproduction in any medium, provided the original work is properly cited. www.e-aaps.org sition flaps as a means of creating a more aesthetic and natural umbilicus.

\section{CASE REPORT}

A 42-year-old female patient had undergone modified radical mastectomy in April 2012 for cancer of the left breast. In May 2014, she underwent delayed breast reconstruction using a transverse rectus abdominis myocutaneous (TRAM) flap. Unfortunately, she subsequently developed full-thickness necrosis of the umbilicus following umbilical transposition and abdominal wall donor-site closure. The area was surgically debrided and directly closed. After 7 months, a vertical scar was present at the surgical site, with no umbilical concavity (Fig. 1).

To reconstruct the umbilicus, four transposition flaps were designed at the site of the umbilical scar. The fact that the surgical scar would be included in the flap was taken into consideration, so the pedicle was set at two points (cephalic and caudal) to ensure there was sufficient blood supply to the flap. Of the four $\mathrm{V}$-shaped flaps, two were designed so that they were horizontal (A and B), and the other two were designed to face in vertical directions (X and Y) (Fig. 2A). The length of the vertical V-shaped flap (X or Y) determines the depth of the umbilicus, while its width determines the size of the umbilical ring (Fig. 2B and 2D). The cut was made 


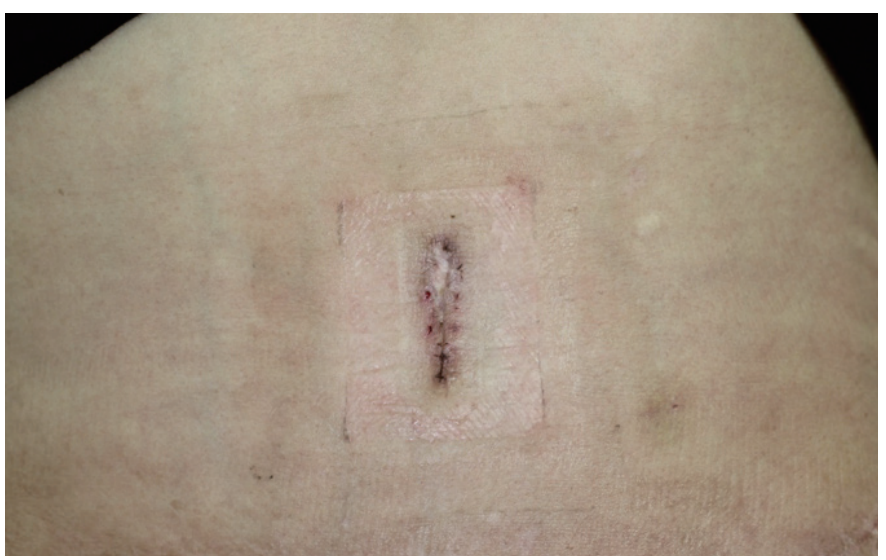

Fig. 1. A case of a 42-year-old woman. Seven months after surgical debridement and direct closure.

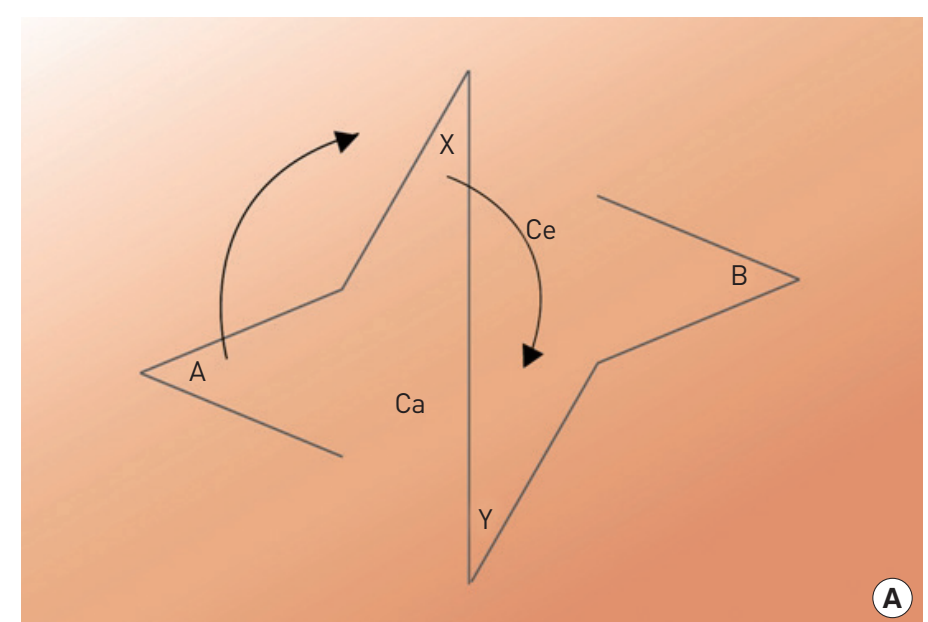

(A)

along the design to allow the four flaps to be completely detached from Scarpa's fascia and elevated. The subcutaneous fat of the vertical flaps was removed (defatting process), the two flaps were folded down, and their tips were firmly anchored to the appropriate location on the linea alba using 4-0 absorbable sutures (Vicryl, Ethicon, Somerville, NJ, USA). To create an aesthetic and verticallyoriented umbilicus, the flap was rotated clockwise and the horizontal flaps (A and B) were transposed on both sides at the sites where the two vertical flaps (X and $\mathrm{Y}$ ) were elevated; the subcutaneous layer was then sutured using 5-0 absorbable sutures (Vicryl, Ethicon, Somerville, NJ, USA). The remaining defects on either side were undermined and directly closed (Fig. 2C). The skin of each flap was closed using simple interrupted sutures with 5-0 non-absorbable nylon sutures (Ethicon, Somerville, NJ, USA) (Fig. 2D

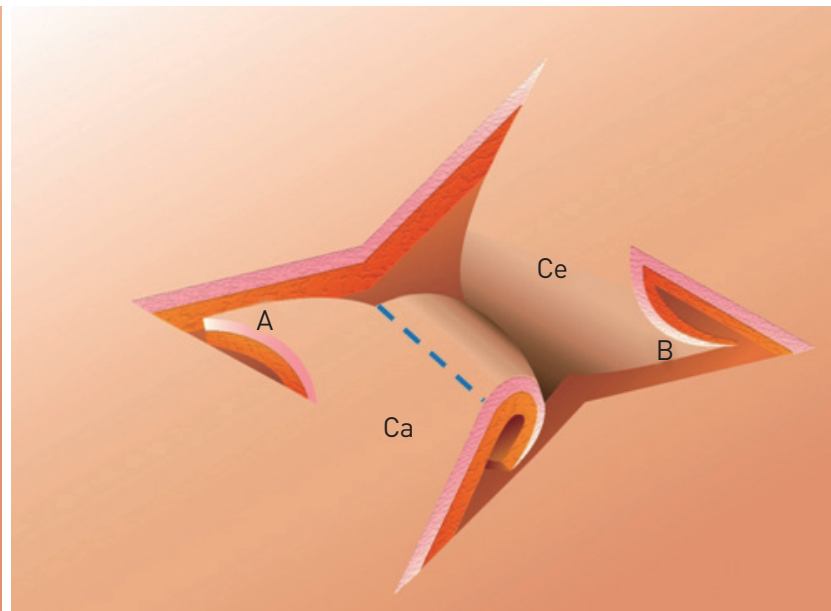

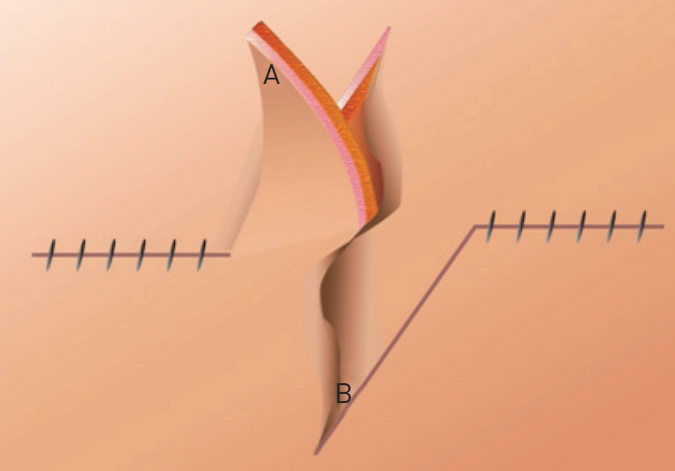

(C)

Fig. 2. Surgical method of four transposition flaps. (A) Design of the four transposition flaps. (B) The flap was elevated and the vertical flaps (X and $Y$ ) were folded down before firmly anchoring onto the linea alba. The horizontal flaps (A and B) were rotated to create a vertical shape. The width of the vertical flap (blue dotted line) determines the size of the umbilical ring. (C) The anchored vertical flap (X and Y) was rotated clockwise and the horizontal flaps ( $A$ and $B$ ) were transposed on both sides at the sites where the two vertical flaps were elevated. The remaining defects were undermined and directly closed. (D) The skin was closed using 5-0 non-absorbable nylon to create the final umbilicus. Ca, caudal flap; Ce, cephalic flap; A, horizontal flap of caudal flap; B, horizontal flap of cephalic flap; X, vertical flap of caudal flap; Y, vertical flap of cephalic flap. 
and 3). The invagination of the umbilicus site was fixed using 4-0 non-absorbable nylon sutures (Ethicon, Somerville, NJ, USA) for bolster dressing. Light compression dressings were used on the surgical site, and the bolster dressing and sutures were removed after 10 days. After all of the stitches were removed, silicone gel (CicaCare $^{\circledR}$; Smith and Nephew, Hull, UK) was applied for scar management.

There were no complications such as flap necrosis, wound infection, hematoma, or disruption. During the 11 months of follow-up, the patient's neoumbilicus maintained a sufficient depth, and the patient was satisfied with the surgical outcome (Fig. 4).

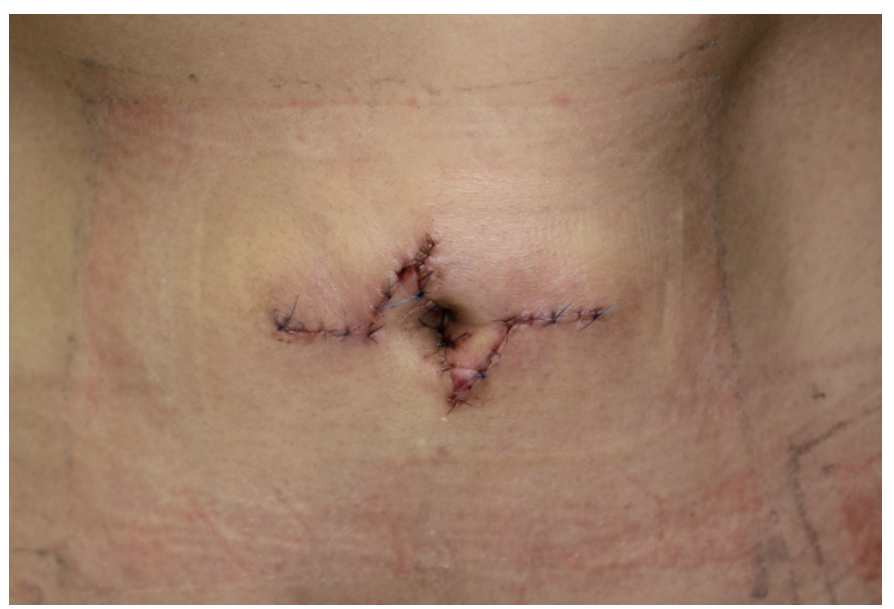

Fig. 3. Immediate postoperative view after umbilical reconstruction. The neoumbilicus shows sufficient depth and a vertical shape before application of the bolster dressing.

\section{DISCUSSION}

The umbilicus is located at the midpoint of the lower abdomen, and is an important aesthetic unit of the abdominal wall. It constitutes the remains of the umbilical cord and its contents, and is a three-dimensional structure consisting of a circular umbilical ring, cylindrical concavity, sulcus, and bottom [5].

The umbilicus as a structure has feminine symbolism, and has significant psychological, physical, and social value. It can also play a role in sexual activity. The absence of the umbilicus due to umbilical necrosis following surgery (for example, due to abdominal surgery) can lead to significant psychological trauma and stress for patients. Innovations in breast reconstruction surgery involve the harvest of tissue from the umbilical area. Meanwhile, the recent increase in the early detection of breast cancer in younger women has led to a higher number of younger breast patients undergoing surgical treatment for breast cancer. Thus, more young women are facing the need for umbilicus reconstruction.

The ideal umbilicus is no larger than $1.5 \mathrm{~cm}$ in width and $2.5 \mathrm{~cm}$ in length, and is vertically oriented with sufficient depth, superior hooding, periumbilical concavity, an inconspicuous scar, and no cicatricial scars [4]. Several surgical techniques for umbilical reconstruction have been developed, including the use of local flaps, pursestring sutures, and cartilage grafts. The most important local flap technique is the inverted $\mathrm{C}-\mathrm{V}$ flap, which showed good short-term results but was associated with long flaps and difficulty in creating umbilici with sufficient depth [1]. Subsequently, the "iris" technique was developed by Miller and Balch [6]. This is a method in which wide excision of the umbilical tissue is performed, followed by the design of four curvilinear incisions and rotation of the four
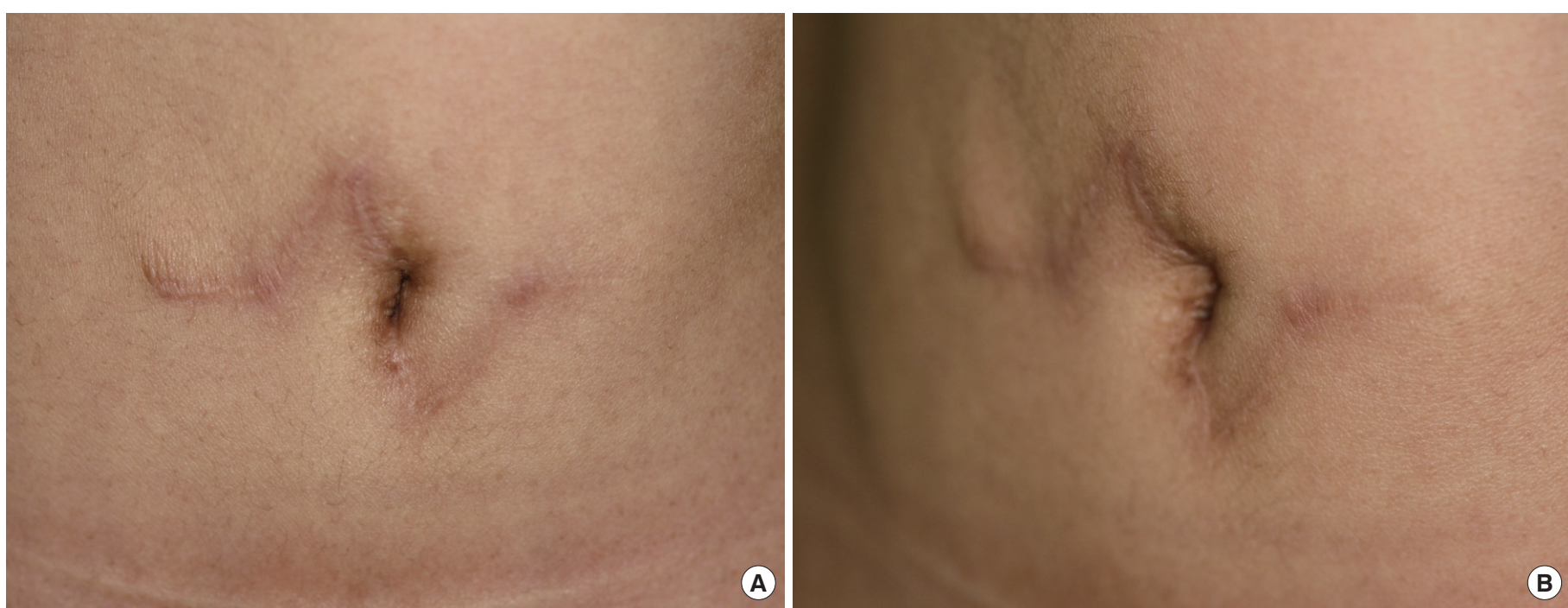

Fig. 4. View at 11 months postoperatively. The results at 11 months after the surgery, demonstrating a natural neoumbilicus with sufficient depth, a vertical shape, and smooth transition of the periumbilical concavity. (A) Anterior-posterior view. (B) Left oblique view. 
flaps to reconstruct the umbilicus. However, one limitation of this method is that it leaves a conspicuous dog-ear scar around the umbilicus. Bartsich and Schwartz [3] have used the purse-string suture method for umbilical reconstruction following abdominal surgical procedures such as recurrent incisional hernia repair or urachal cyst repair. However, this method leaves open wounds and poses difficulties for wound management. The cartilage graft method was introduced to create sufficient depth for the umbilicus, but requires a donor site and is technically challenging to perform [7]. Despite these various techniques, there has been no optimal umbilical reconstruction method developed to date.

In this study, the site of umbilical necrosis was surgically debrided and the reconstruction of the umbilicus was performed approximately 7 months following surgical debridement to stabilize the wound. Compared to previously reported umbilical reconstruction methods using local flaps, there are several differences in the method developed in this study using four transposition flaps. When the four transposition flaps were designed, the tips of the two vertical flaps (X and Y) were firmly anchored to the linea alba to create sufficient umbilical depth. We also created the largest possible flap with minimal scarring by taking into consideration the absorption afterwards. The flap pedicle was placed at two points that were superior and inferior to ensure sufficient blood supply to the flap (as the flap was elevated from the previous scar tissue) and to minimize absorption and flap necrosis. Lastly, the flap was rotated to create a vertically-oriented and aesthetically pleasing umbilicus, and to create a periumbilical concavity that allows for a smooth transition from the abdominal wall to the umbilicus.

Our technique using four transposition flaps is simple to design and is not difficult to perform. The technique allows for maintenance of sufficient umbilical depth, and the creation of an aesthetically-pleasing, vertically-oriented umbilicus. The flap is well-vas- cularized, and the previous scar does not need to be removed before umbilical reconstruction, which means that the previous scar can be hidden inside the umbilicus. This case shows that umbilical reconstruction using a four transposition flap in patients with the loss of the umbilicus from various causes is a safe and reproducible method. Further follow-up and long-term data will add to our understanding of the value of this surgical method of umbilical reconstruction.

\section{PATIENT CONSENT}

Patients provided written consent for the use of their images.

\section{REFERENCES}

1. Hong YG, Cho JJ. Reconstruction of scarred umbilicus using an inverted C-V flap: a case report. J Korean Soc Plast Reconstr Surg 2007; 34:653-5.

2. Pfulg M, Van de Sijpe K, Blondeel P. A simple new technique for neoumbilicoplasty. Br J Plast Surg 2005;58:688-91.

3. Bartsich SA, Schwartz MH. Purse-string method for immediate umbilical reconstruction. Plast Reconstr Surg 2003;112:1652-5.

4. Rozen SM, Redett R. The two-dermal-flap umbilical transposition: a natural and aesthetic umbilicus after abdominoplasty. Plast Reconstr Surg 2007;119:2255-62.

5. Park ES, Kim MS, Kim YB. Immediate umbilical reconstruction for urachal cancer. J Korean Soc Aesthetic Plast Surg 2003;9:59-62.

6. Miller MJ, Balch CM. "Iris" technique for immediate umbilical reconstruction. Plast Reconstr Surg 1993;92:754-6.

7. Matsuo K, Kondoh S, Hirose T. A simple technique for reconstruction of the umbilicus, using a conchal cartilage composite graft. Plast Reconstr Surg 1990;86:149-51. 